\title{
Analysis of the defect clusters in congruent lithium tantalate
}

\author{
Anastasia Vyalikh, ${ }^{1, *}$ Matthias Zschornak, ${ }^{1,2}$ Thomas Köhler, ${ }^{1}$ Melanie Nentwich, ${ }^{1}$ Tina Weigel, ${ }^{1}$ Juliane Hanzig, \\ Ruslan Zaripov, ${ }^{3}$ Evgenia Vavilova, ${ }^{3}$ Sibylle Gemming, ${ }^{2,4}$ Erica Brendler, ${ }^{5}$ and Dirk C. Meyer ${ }^{1}$ \\ ${ }^{1}$ Institut für Experimentelle Physik, Technische Universität Bergakademie Freiberg, Leipziger Str. 23, Freiberg, Germany \\ ${ }^{2}$ Institute of Ion Beam Physics and Materials Research, Helmholtz-Zentrum Dresden-Rossendorf, PO Box 510119, Dresden, Germany \\ ${ }^{3}$ Kazan E. K. Zavoisky Physical-Technical Institute of the RAS, Sibirsky Tract, 10/7 420029 Kazan, Russia \\ ${ }^{4}$ Institute of Physics, Technische Universität Chemnitz, Chemnitz, Germany \\ ${ }^{5}$ Institut für Analytische Chemie, Technische Universität Bergakademie Freiberg, 09596 Freiberg, Germany
}

(Received 15 August 2017; published 11 January 2018)

\begin{abstract}
A wide range of technological applications of lithium tantalate (LT) is closely related to the defect chemistry. In literature, several intrinsic defect models have been proposed. Here, using a combinational approach based on DFT and solid-state NMR, we demonstrate that distribution of electric field gradients (EFGs) can be employed as a fingerprint of a specific defect configuration. Analyzing the distribution of ${ }^{7} \mathrm{Li}$ EFGs, the FT-IR and electron spin resonance (ESR) spectra, and the ${ }^{7} \mathrm{Li}$ spin-lattice relaxation behavior, we have found that the congruent LT samples provided by two manufacturers show rather different defect concentrations and distributions although both were grown by the Czochralski method. After thermal treatment hydrogen out-diffusion and homogeneous distribution of other defects have been observed by ESR, NMR, and FT-IR. The defect structure in one of two congruent LT crystals after annealing has been identified and proved by defect formation energy considerations, whereas the more complex defect configuration, including the presence of extrinsic defects, has been suggested for the other LT sample. The approach of searching the EFG fingerprints from DFT calculations in NMR spectra can be applied for identifying the defect clusters in other complex oxides.
\end{abstract}

DOI: 10.1103/PhysRevMaterials.2.013804

\section{INTRODUCTION}

Crystals of lithium tantalate $\mathrm{LiTaO}_{3}$ (LT) and lithium niobate $\mathrm{LiNbO}_{3}(\mathrm{LN})$ are currently commercially used for integrated optics because of their excellent electro-optical and nonlinear optical properties [1,2]. These materials are also known for their ferroelectric, piezoelectric, pyroelectric, and acoustic properties that result in various technological applications. Novel interesting applications of LT and LN are water disinfection and the generation of reactive oxygen species employing the pyroelectric effect [3] and high-efficiency frequency doubling at telecommunication wavelengths proposed as an alternative for silicon photonics [4].

Congruent LT and LN crystals grown by the Czochralski method have a Li deficiency as compared to the stoichiometric crystal structures $[5,6]$. As a result they contain a number of intrinsic point defects such as $\mathrm{Li}$ vacancies or $\mathrm{Ta}(\mathrm{Nb})$ antisite defects. Since these defects are charged, further defects with countercharges are required in order to guarantee overall charge neutrality. Thus, for energetic reasons, clusters of closely spaced defects are favored and considered in literature (Fig. 1) [7-10].

Much effort has been made in the past in order to characterize the defect structure in lithium niobate, which is isomorphic

\footnotetext{
*Anastasia.Vyalikh@physik.tu-freiberg.de

Published by the American Physical Society under the terms of the Creative Commons Attribution 4.0 International license. Further distribution of this work must maintain attribution to the author(s) and the published article's title, journal citation, and DOI.
}

to lithium tantalate. The most widely accepted model proposed by Lerner et al. [10] and extended by Kim et al. [11] comprises a $\mathrm{Nb}$-antisite defect surrounded by three lithium vacancies in the nearest neighborhood and by a fourth $\mathrm{Li}$ vacancy located along the polar $c$ axis [referred hereafter as model M1, Fig. 1(b)]. M1 has been preferred based on real-time domain studies [11], combined x-ray and neutron powder diffraction studies $[9,12]$, nuclear magnetic resonance (NMR) [13], and computer-simulation studies of intrinsic defects [14]. An alternative model with a $\mathrm{Nb}$ on $\mathrm{Li}$ sites compensated by additional Nb-site vacancies (M2) has been suggested based on ${ }^{93} \mathrm{Nb}$ NMR [15] and x-ray single-crystal diffraction data [16], but compromised later by Zotov [9] and Iyi [12] based on revised structural refinements. In model M3 [Fig. 1(c)], all the excess $\mathrm{Nb}$ amount is in the vacant octahedron, which is highlighted in gray in Fig. 1(a). Model M4 consists of one $\mathrm{Nb}$ on a $\mathrm{Li}$ site, and, in contrast to $\mathrm{M} 1$, one $\mathrm{Li}$ on a $\mathrm{Nb}$ site [17]. This model cannot account for the nonstoichiometry but may appear in conjunction with other defect models as stable defect. Model M5 has been proposed on the basis of stereochemical considerations representing a combination of the models M1 and M3 with a random distribution of the excess $\mathrm{Nb}$ atoms on both $\mathrm{Li}$ and vacancy sites [18]. In model M6, a lithium atom is placed on an interstitial position, while the excess amount of $\mathrm{Nb}$ occupies vacant octahedra. Comprehensive experimental verifications and theoretical calculations have not resulted in a sole defect model for congruent lithium niobate.

Even less is known for lithium tantalate, which is the subject of the present study. The crystal structures of stoichiometric LT and LN were first published by Abrahams et al. [19-22]. The cation concentration ratios of congruently grown 


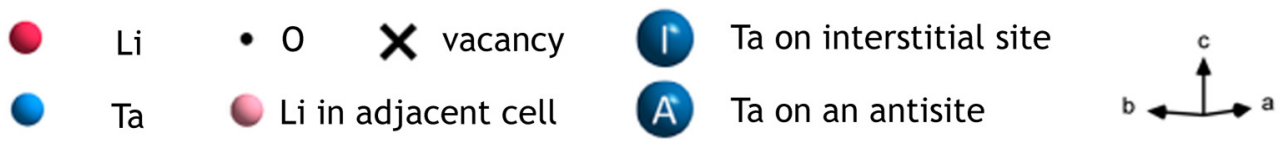

(a) Stoichiometric LT

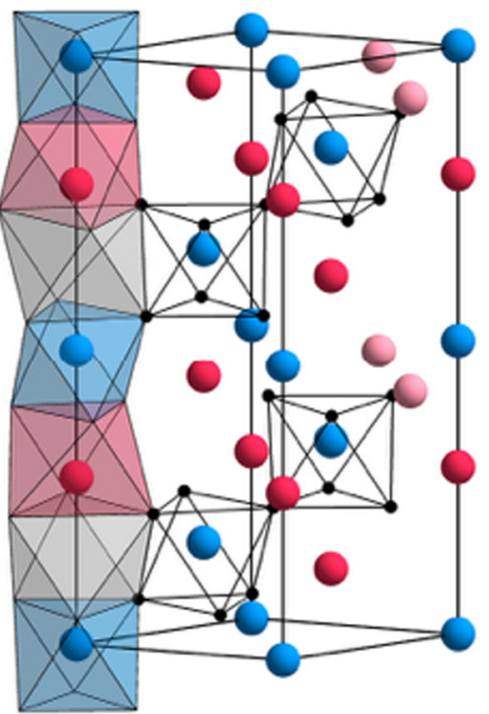

(b) $M 1$

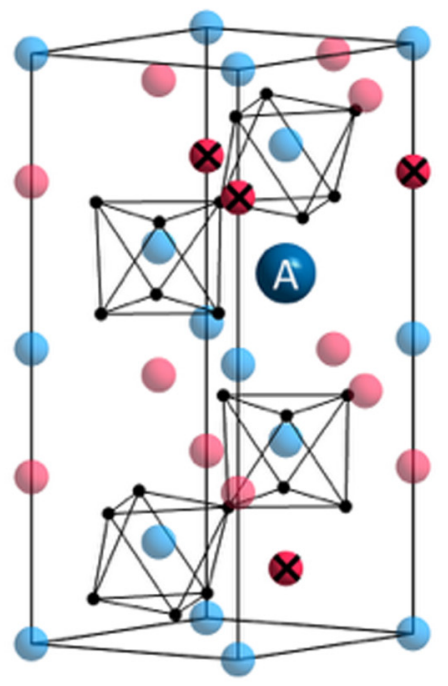

(c) $M 3$

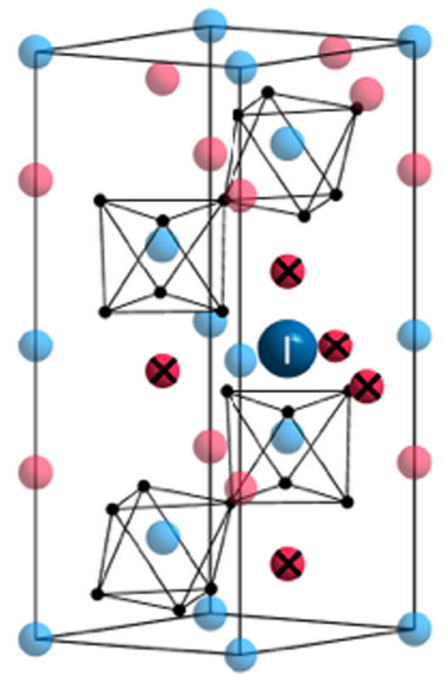

FIG. 1. Crystal structures of (a) stoichiometric, defect-free lithium tantalate, (b) M1, and (c) M3 defect cluster models. In the defect-free LT, the vacant octahedra are highlighted in gray to visualize the oxygen partial structure. Crosses and large dark blue spheres denote modified atom positions in the defect cluster models.

compositions deviate slightly, yielding $[\mathrm{Li}] /[\mathrm{Nb}]=48.5 / 51.5$ and $[\mathrm{Li}] /[\mathrm{Ta}]=48.75 / 51.25$ for $\mathrm{LN}$ and $\mathrm{LT}$, respectively [5,6]. Thus, Li deficiency and the structural isomorphism of LT and LN allows us to assume that the defect models proposed in literature for LN can be considered for LT. However, our recent investigations of lithium tantalate and lithium niobate using light polarization resolved FT-IR spectroscopy have showed that these materials can be characterized by different defect concentrations and intrinsic defect clusters, which are besides preferably decorated by hydrogen [23].

Solid-state NMR, which focuses on the short-range order, is ideally suited to probe local changes at lattice defects and structural imperfections in crystalline solids [24]. Moreover, NMR on quadrupolar nuclei, which possess nonzero electric quadrupole moments, such as ${ }^{7} \mathrm{Li}(I=3 / 2)$ and ${ }^{93} \mathrm{Nb}(I=$ $9 / 2$ ), is particularly sensitive to the presence of electric field gradients (EFGs) at the nucleus. Thus, this technique allows obtaining information about the local changes at defect sites in LT and LN. The crystal structures in LN and LT have been studied using solid-state NMR in a number of works [13,15,25-30]. Halstead [27] investigated atomic motions in a single crystal of lithium niobate on the basis of temperaturedependent ${ }^{7} \mathrm{Li}$ NMR spectra. He suggested that the observed satellite lines are the superpositions of lines with different quadrupole splitting, which arise from $\mathrm{Li}$ atoms in different regions of the crystal. Peterson and Carnevale [15] observed an additional line in the ${ }^{93} \mathrm{Nb}$ NMR spectrum of congruent $\mathrm{LN}$, which comprised $6 \%$ of the total spectral intensity. Initially, this line was attributed to $\mathrm{Nb}$ atoms occupying the $\mathrm{Li}$ sites that corresponds to the M2 defect model. Later, a careful reexamination based on calculating the probability distribution of EFG components [31] revealed a more complex origin of the subsidiary absorptions observed in Ref. [15]. Additional lines of weaker intensity and of large quadrupolar splitting in the ${ }^{7} \mathrm{Li}$ and ${ }^{93} \mathrm{Nb}$ NMR spectra have been reported in Ref. [30] for two of four congruent LN samples of different origin. Calculations of the ${ }^{93} \mathrm{Nb}$ EFG therein for the M1 and M2 defect models, as well as for the oxygen vacancy model could not explain the appearance of the weak lines in the NMR spectra. Therefore, it was suggested that the appearance of the additional lines is linked to the crystal growth technologies, which may cause the appearance of further phases, such as $\mathrm{Nb}_{2} \mathrm{O}_{5}$ or $\mathrm{LiNb}_{3} \mathrm{O}_{8}$. Blümel et al. [28] did not observe additional signals, which could account for $5 \%$ of wrongly positioned $\mathrm{Nb}$ atoms assumed in model M2. Based on their observations, the authors suggested that either model M1 is favorable for LN, or, neither defect model should be featured in the NMR spectra [28].

Thus, despite continued interest in LN and LT due to their technological importance, the open question remains in understanding their defect cluster structure. The interest is boosted by the lack of theoretical and experimental data, which concern the defect models in LT. At present, the advances in computational software and hardware offer new opportunities to calculate large unit cells required for these defect clusters. In the present work, we therefore attempt to inspect the defect model structures in LT using a combination of theoretical and experimental methods previously employed in the studies of LN.

We have performed electronic structure calculations with density functional theory (DFT) to assess the stability of the LT defect structures from first principles. Furthermore, EFGs at Li sites have been computed in order to reveal whether EFGs may 
TABLE I. Defect clusters, their concentrations and defect formation energies for different defect models in LT. The symbols follow the Kröger-Vink notation $X_{Y}^{Z}$, where the element $X$ is at a position of former element $Y$ with relative charge $Z(\bullet-$ positive charge, ' - negative charge), and $V$ and i denote vacant and interstitial sites.

\begin{tabular}{|c|c|c|c|c|c|}
\hline Model & Central defect & $\begin{array}{c}\text { Compensating } \\
\text { defect }\end{array}$ & Reaction & $\begin{array}{c}\text { Defect } \\
\text { concentration }^{\mathrm{b}}\end{array}$ & $\begin{array}{l}\text { Formation } \\
\text { energy, eV }\end{array}$ \\
\hline M1 & $\mathrm{Ta}_{\mathrm{Li}}^{\bullet \bullet \bullet \bullet ~}$ & $4 V_{\mathrm{Li}}^{\prime}$ & $3 \mathrm{Ta}_{2} \mathrm{O}_{5} \rightarrow 5 \mathrm{Ta}_{\mathrm{Ta}}^{\times}+15 \mathrm{O}_{\mathrm{O}}^{\times}+\left(1 \mathrm{Ta}_{\mathrm{Li}}^{\bullet \bullet \bullet}+4 V_{\mathrm{Li}}^{\prime}\right)$ & $1 / 16$ & +1.94 \\
\hline M2 & $5 \mathrm{Ta}_{\mathrm{Li}}^{\bullet \cdots \bullet \bullet}$ & $4 V_{\mathrm{Ta}}^{,, \cdots}$ & $3 \mathrm{Ta}_{2} \mathrm{O}_{5} \rightarrow 1 \mathrm{Ta}_{\mathrm{Ta}}^{\times}+15 \mathrm{O}_{\mathrm{O}}^{\times}+\left(5 \mathrm{Ta}_{\mathrm{Li}}^{\bullet \bullet \bullet \bullet}+4 V_{\mathrm{Ta}}^{,, \cdots,}\right)$ & $1 / 16$ & +10.90 \\
\hline $\begin{array}{l}\text { M3 } \\
\text { M3-1 } \\
\text { M3-2 } \\
\text { M3-3 }\end{array}$ & $\mathrm{Ta}_{\mathrm{i}}^{\bullet \bullet \bullet \bullet \bullet}$ & $\begin{array}{l}5 V_{\mathrm{Li}}^{,} \\
V_{\mathrm{Ta}}^{,,,,}\end{array}$ & $\begin{aligned} 3 \mathrm{Ta}_{2} \mathrm{O}_{5} & \rightarrow 5 \mathrm{Ta}_{\mathrm{Ta}}^{\times}+15 \mathrm{O}_{\mathrm{O}}^{\times}+\left(1 \mathrm{Ta}_{i}^{\bullet \bullet \bullet \bullet}+5 V_{\mathrm{Li}}^{\prime}\right) \\
1 \mathrm{LiTaO}_{3} & \rightarrow 1 \mathrm{Li}_{\mathrm{Li}}^{\times}+3 \mathrm{O}_{\mathrm{O}}^{\times}+\left(1 \mathrm{Ta}_{i}^{\bullet \bullet \bullet \bullet}+6 V_{\mathrm{Ta}}^{, \cdots, \cdots}\right)\end{aligned}$ & $\begin{array}{l}1 / 16 \\
- \\
- \\
-\end{array}$ & $\begin{array}{l}+1.61 \\
+4.14 \\
+5.78 \\
+4.65\end{array}$ \\
\hline $\begin{array}{l}\text { M4- } 1^{\mathrm{a}} \\
\text { M4-2 } \\
\text { M } 4-3^{\mathrm{a}}\end{array}$ & $\mathrm{Ta}_{\mathrm{Li}}^{\bullet \bullet \bullet \bullet ~}$ & $\mathrm{Li}_{\text {Ta }}^{\prime,}$ & $1 \mathrm{LiTaO}_{3} \rightarrow 3 \mathrm{O}_{\mathrm{O}}^{\times}+\left(1 \mathrm{Ta}_{\mathrm{Li}}^{\bullet \cdots \bullet}+1 \mathrm{Li}_{\mathrm{Ta}}^{\mathrm{\prime}, \prime \prime}\right)$ & $\begin{array}{l}- \\
-\end{array}$ & $\begin{array}{l}+3.01 \\
+3.75 \\
+4.61\end{array}$ \\
\hline $\begin{array}{l}\text { M5 } \\
\text { M5-1 } \\
\text { M5-2 } \\
\text { M5-3 } \\
\text { M5-4 } \\
\text { M5-5 }\end{array}$ & $\mathrm{Ta}_{\mathrm{Li}}^{\bullet \bullet \bullet \bullet}, \mathrm{Ta}_{\mathrm{i}}^{\bullet \bullet \bullet \bullet \bullet ~}$ & $\begin{array}{c}9 V_{\mathrm{Li}}^{,} \\
4 V_{\mathrm{Li}}^{,}, V_{\mathrm{Ta}}^{,,,},\end{array}$ & $\begin{array}{c}6 \mathrm{Ta}_{2} \mathrm{O}_{5} \rightarrow 10 \mathrm{Ta}_{\mathrm{Ta}}^{\times}+30 \mathrm{O}_{\mathrm{O}}^{\times}+\left(1 \mathrm{Ta}_{\mathrm{Li}}^{\bullet \bullet \bullet}+1 \mathrm{Ta}_{i}^{\bullet \bullet \bullet \bullet}+9 V_{\mathrm{Li}}^{\prime}\right) \\
3 \mathrm{Ta}_{2} \mathrm{O}_{5} \rightarrow 4 \mathrm{Ta}_{\mathrm{Ta}}^{\times}+15 \mathrm{O}_{\mathrm{O}}^{\times}+\left(1 \mathrm{Ta}_{\mathrm{Li}}^{\bullet \cdots \bullet \bullet}+1 \mathrm{Ta}_{i}^{\bullet \bullet \bullet \bullet \bullet}+4 V_{\mathrm{Li}}^{\prime}+1 V_{\mathrm{Ta}}^{,,, \prime}\right)\end{array}$ & $\begin{array}{l}1 / 32 \\
1 / 16 \\
1 / 16 \\
1 / 16 \\
1 / 16 \\
1 / 16\end{array}$ & $\begin{array}{l}+7.16 \\
+1.94 \\
+1.72 \\
+1.55 \\
+1.57 \\
+1.74\end{array}$ \\
\hline M6 & $\mathrm{Li}_{\mathrm{i}}^{\bullet}, \mathrm{Ta}_{\mathrm{i}}^{\bullet \cdots \bullet \bullet}$ & $6 V_{\mathrm{Li}}^{\prime}$ & $3 \mathrm{Ta}_{2} \mathrm{O}_{5}+1 \mathrm{LiTaO}_{3} \rightarrow 6 \mathrm{Ta}_{\mathrm{Ta}}^{\times}+18 \mathrm{O}_{\mathrm{O}}^{\times}+\left(1 \mathrm{Li}_{i}^{\bullet}+1 \mathrm{Ta}_{i}^{\bullet \bullet \bullet \bullet}+6 V_{\mathrm{Li}}^{\prime}\right)$ & $1 / 16$ & +7.03 \\
\hline
\end{tabular}

aThese submodels cannot account for the nonstoichiometry but may appear in conjunction with other defect models as stable defect in congruent LT.

${ }^{\mathrm{b}}$ Defect concentration $(1 / n)$ is estimated to account for the non-stoichiometry in congruent LT, where $n$ is a number of unit cells containing one defect complex.

be regarded as a fingerprint of a specific defect configuration [32]. For experimental verification, ${ }^{7} \mathrm{Li}$ solid-state NMR has been applied, which provides information on the parameters of ${ }^{7} \mathrm{Li}$ quadrupole coupling tensor, from which EFGs can be directly calculated.

For defect characterization in congruent LT single crystals, electron spin resonance (ESR) and Fourier transform infrared (FT-IR) spectroscopies have been applied. ESR enables us to detect paramagnetic centers, such as metal impurities, localized charge carriers, F-type centers, broken chemical bonds, and $\mathrm{Ta}^{4+}$ ions. FT-IR is sensitive to the concentration of hydroxide groups and allows quantitative characterization of the crystal stoichiometry based on the position and broadening of $\mathrm{OH}^{-}$stretching vibrations $[23,33]$.

Assuming that the actual defect structure of congruently molten crystals can vary between the samples from different sources, and moreover, can be decisively affected by subsequent sample treatment, we have investigated two as-grown crystals of different origin. Furthermore, both samples were subjected to thermal treatment by vacuum annealing, because this procedure is commonly used to remove unwanted defects and to redistribute the defects more homogeneously. To ensure comparability, the treatment and characterization has been employed by the same set of techniques.

\section{METHODS}

\section{A. Theoretical simulations}

DFT calculations were performed to evaluate the stability of several defect models and to interpret the experimental ${ }^{7}$ Li NMR spectra comparing to theoretical EFG values. The models for DFT calculations were created assuming that one central defect forces the structure to change, whereas the charge is compensated by adding other defects to energetically stabilize the defect cluster. Table I summarizes the defect cluster models representing the central and compensating defects in Kröger-Vink notation as well as the reaction equations liable for the defect cluster [34] and the defect concentrations per crystallographic LT unit cell to account for Li deficiency of about $5 \%$. Figure 1 shows the crystal structures of stoichiometric (defect-free) LT and the defect structures corresponding to M1 and M3 models. The central defects of models M3 and M5 as proposed by Zotov et al. [9] allow in addition alternative electrical compensation by one Ta vacancy instead of five Li vacancies. These substitutes enable different local arrangements of the charge compensating defects, varying by the distance to each other. Therefore, models M3 and M5 have been extended to three and five additional defect models, denoted as M3-x and M5-x, respectively. Model M4 also offers three possibilities of arrangement concerning the distance of both defects to each other yielding M4-x submodels [35].

For DFT electronic structure calculations and computation of EFGs at the Li sites, the projector-augmented wave (PAW) method [36] in PBE parametrization [37] as implemented in the VASP code [38] was employed. Total energy calculations converged better than $10^{-7} \mathrm{eV}$ with a maximum kinetic energy of $450 \mathrm{eV}$ for the plane wave basis set. A Hubbard- $U$ correction for the Ta $d$ states with an effective Dudarev potential of $7 \mathrm{eV}$ was applied. As $k$-point grids $\Gamma$-centered MonkhorstPack meshes equivalent to a $12 \times 12 \times 4$ sampling of the LT cell with spacings less than $0.02 \times 2 \pi \AA^{-1}$ were chosen. The defect clusters were incorporated in $2 \times 2 \times 1$ supercells with the cell parameters fixed to the defect-free fully relaxed LT parameters. Atomic positions within the defect structures 
relaxed to forces less than $10^{-3} \mathrm{eV} \AA^{-1}$ prior to final total energy and EFG calculations. To account for stoichiometry variations, defect formation energy for the defect structure $M$

$$
E_{f}(M)=E^{\mathrm{tot}}(M)-x \cdot E^{\mathrm{tot}}(\mathrm{LT})-y \cdot E^{\mathrm{tot}}\left(\mathrm{Ta}_{2} \mathrm{O}_{5}\right)
$$

has been normalized to the defect-free LT structure $(R 3 c)$ and $\mathrm{Ta}_{2} \mathrm{O}_{5}(P m m 2)$ with respective total energies $E^{\text {tot }}(\mathrm{LT})$ and $E^{\text {tot }}\left(\mathrm{Ta}_{2} \mathrm{O}_{5}\right)$, whereas $x$ and $y$ are chosen to represent the particular number of atoms in the supercell accordingly.

The corresponding formulas for computing the defect formation energies for all models are given in Ref. [35]. Convergence of formation energies with respect to finite supercell size has been checked on models M5 and M6 in doubling the supercell volume. The results yield a systematic reduction of about $10 \%$ due to further decrease of elastic energy. In addition, to analyze the effect of different defect concentrations on the quadrupolar coupling constant, EFGs for one defect cluster within an extended $2 \times 2 \times 2$ supercell (in models M5 and M6) and for two defect clusters within the $2 \times 2 \times 1$ supercell (in models M1, M5, and M6) were calculated with fixed cell geometry. The two defect clusters were arranged in such a way that their centers of charge have the shortest distance. For comparison with the experimental NMR data, the calculated EFGs were converted into the ${ }^{7} \mathrm{Li}$ quadrupolar coupling constants $C_{Q}$ and asymmetry parameters $\eta$ using a value of the nuclear quadrupole moment $\mathrm{Q}\left({ }^{7} \mathrm{Li}\right)=40.0(3) \mathrm{mb}$ given in Ref. [39] as described in Ref. [35].

\section{B. Experimental part}

\section{Sample preparation and characterization}

The LT samples were commercial thin plates grown by the Czochralski method. Nonstoichiometry of both samples LT-A (CrysTec GmbH, Berlin, Germany) and LT-B (EPCOS AG, Munich, Germany) was estimated to be $[\mathrm{Li}] /[\mathrm{Ta}]=48.7 / 51.3$ using FT-IR. The LT-A sample was a double-side polished crystal plate of $5 \times 5 \times 0.5 \mathrm{~mm}^{3}$ size and was used without treatment. Two crystals of $30 \times 10 \mathrm{~mm}^{2}$ size were cut out of a one-side polished wafer from EPCOS and polished to a thickness of $500 \mu \mathrm{m}$ using $\mathrm{SiC}$ sandpaper, followed by polishing with diamond suspension on both sides. One plate was used without further processing (LT-B). The second plate and another CrysTec-crystal were subjected to thermal treatment by heating in a vacuum oven at $550{ }^{\circ} \mathrm{C}$ for 32 hours. The treated samples with slight but visible darkening were termed LT-A-t and LT-B-t, respectively. Details of the crystal orientation are given in Ref. [35]. The crystal surfaces were cleaned in ethanol before each measurement.

\section{2. ${ }^{7} \mathrm{Li} N M R$}

Static ${ }^{7} \mathrm{Li}$ NMR measurements were performed on a Bruker Avance III $400 \mathrm{MHz}$ spectrometer operating at a resonance frequency of $155.5 \mathrm{MHz}$ using a static probe head (NMR Service GmbH, Erfurt). Single crystals were mounted in a twin-axis goniometer with the crystal $c$ axis parallel to the direction of the external magnetic field and placed into an $8 \mathrm{~mm}$ solenoid coil. A polyether ether ketone (PEEK) cover shell of the goniometer enabled protection of the single crystals from the piezoelectric ringing. Static ${ }^{7} \mathrm{Li}$ NMR spectra were recorded using a solid echo pulse sequence with a pulse duration of $3.3 \mu \mathrm{s}$, a pulse delay of $96 \mu \mathrm{s}$ and 64 repetitions. For measurement of LT-A a frequency step of $300 \mathrm{kHz}$ was applied to cover the whole spectrum of $2 \mathrm{MHz}$ in width. For orientational dependence, the sample was rotated in the $a b$ plane with the crystal $c$ axis fixed parallel to the direction of the magnetic field. All measurements were performed at room temperature. The spectra were fitted using DMFIT [40]. ${ }^{7} \mathrm{Li}$ NMR spin-lattice relaxation times were measured on the central line by a standard saturation recovery pulse sequence employing a saturation pulse train of 50 pulses separated by $0.2 \mathrm{~ms} .22$ increments in the range from $0.01-800 \mathrm{~s}$ and eight repetitions were used. The magnetization build-up profiles were analyzed with a saturation recovery function for a spin$3 / 2$ nucleus at the central transition.

\section{3. $E S R$}

ESR spectra were recorded on a Bruker EMXplus spectrometer at a frequency of $9.4 \mathrm{GHz}$ (X band). The samples were glued to a quartz rod, inserted into a cylindrical superhigh-sensitivity probe and measured at the orientation of the crystal $c$ axis parallel to the magnetic field. The angle was controlled by the ER218PG1 Programmable Goniometer (Bruker). All measurements were performed at a $20 \mathrm{~mW}$ microwave power and a temperature of $10 \mathrm{~K}$ using the ESR900 cryostat and the temperature controller ITC503. Contributions from the resonator, the sample holder, and glue were measured independently and found to be negligible. Baseline correction as well as normalization to the sample weight were applied.

\section{FT-infrared spectroscopy}

Fourier transform infrared (FT-IR) spectra were obtained using a Tensor 27 Bruker infrared spectrometer at room temperature. The measurements were performed in the range of $1250-4000 \mathrm{~cm}^{-1}$ at a resolution of $3 \mathrm{~cm}^{-1}$. Transmission measurements were performed in the direction parallel to the crystallographic $c$ axis. A KRS-5 thallium iodide bromide infrared polarizer with a polarization of $99 \%$ was employed to measure the maximal stretching vibration under the same conditions with respect to the $c$ axis in all samples, because of the anisotropic nature of the $\mathrm{OH}^{-}$stretching vibration [23].

\section{RESULTS}

\section{A. DFT calculations}

The calculated formation energies presented in Table I spread significantly demonstrating different stability of the proposed defect structures. Models M1, M3, and M5-x are found to be most energetically favorable for defect formation due to their formation energies below $2 \mathrm{eV}$. Models M3-x and M4-x demonstrate energies of about twice larger, offering a reasonable possibility to be present in as grown LT crystals at room temperature. Models M5 and M6 show formation energies of about $7 \mathrm{eV}$ and are considered unlikely in LT. Model M2 with more than $10 \mathrm{eV}$ for the supercell is least favorable of all defects.

First, the case of one defect cluster within a computational cell comprised of $2 \times 2 \times 1$ unit cells has been considered for calculating EFGs at all Li sites. The calculated EFGs were converted into the ${ }^{7} \mathrm{Li}$ quadrupolar coupling constants $C_{Q}$, 


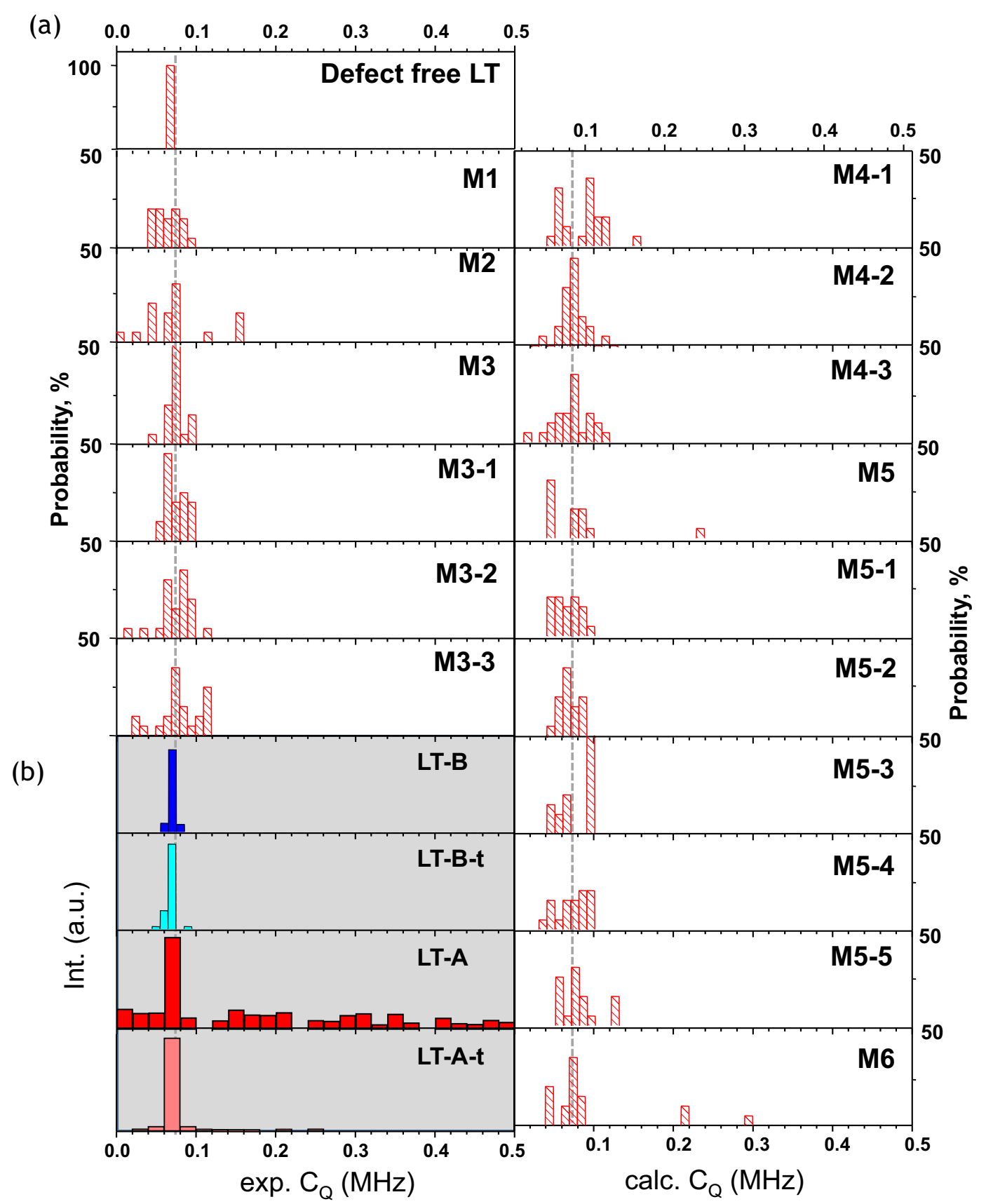

FIG. 2. (a) (white background) Classification of calculated quadrupolar coupling constants $C_{Q}$ for different defect models. (b) (gray background) Classification of experimental quadrupolar coupling constants for the LT samples. Note: Class size is doubled for LT A and LT-A-t for better visualization of minor signals. The dashed vertical line denotes the position of the dominant satellite in the experimental spectra.

which were then sorted into bins of a frequency width of $0.01 \mathrm{MHz}$ on the classification diagrams. The results for defectfree LT and 16 defect clusters are presented in Fig. 2(a). For the defect-free LT crystal structure a $C_{Q}$ of $78.0 \mathrm{kHz}$ has been calculated, which agrees well with literature [26]. As expected for threefold rotational site symmetry of Li positions in LT, the asymmetry parameter is zero. The dominant $C_{Q}$ contribution in all defect models appears at approximately $0.07 \mathrm{MHz}$, which is close to that in defect-free LT. Additional signals of smaller intensity are calculated within the $C_{Q}$ range between 0.04 and 0.12 MHz. For models M2, M4-1, M5, M5-5, and M6, further minor signals are observed at $C_{Q}$ of about $0.3 \mathrm{MHz}$. Analysis of the asymmetry parameters (not presented here) also shows their variability depending on the defect structure and $\mathrm{Li}$ location in respect to the defect. The calculated data show unambiguously that the various defect models demonstrate different distributions of EFGs that can be regarded as a fingerprint of a specific defect configuration. Further, with sufficient spectral resolution, such EFG fingerprints can be recognized in the NMR spectra.

As further dilution is necessary to approach the realistic system with $5 \%$ Li deficiency for all nonstoichiometric defect clusters considered above, we have varied the boundary conditions for certain defect models. On the other hand, increasing the number of the defects in the cell allows us to simulate the case of the defect agglomeration. This approach enabled us to 

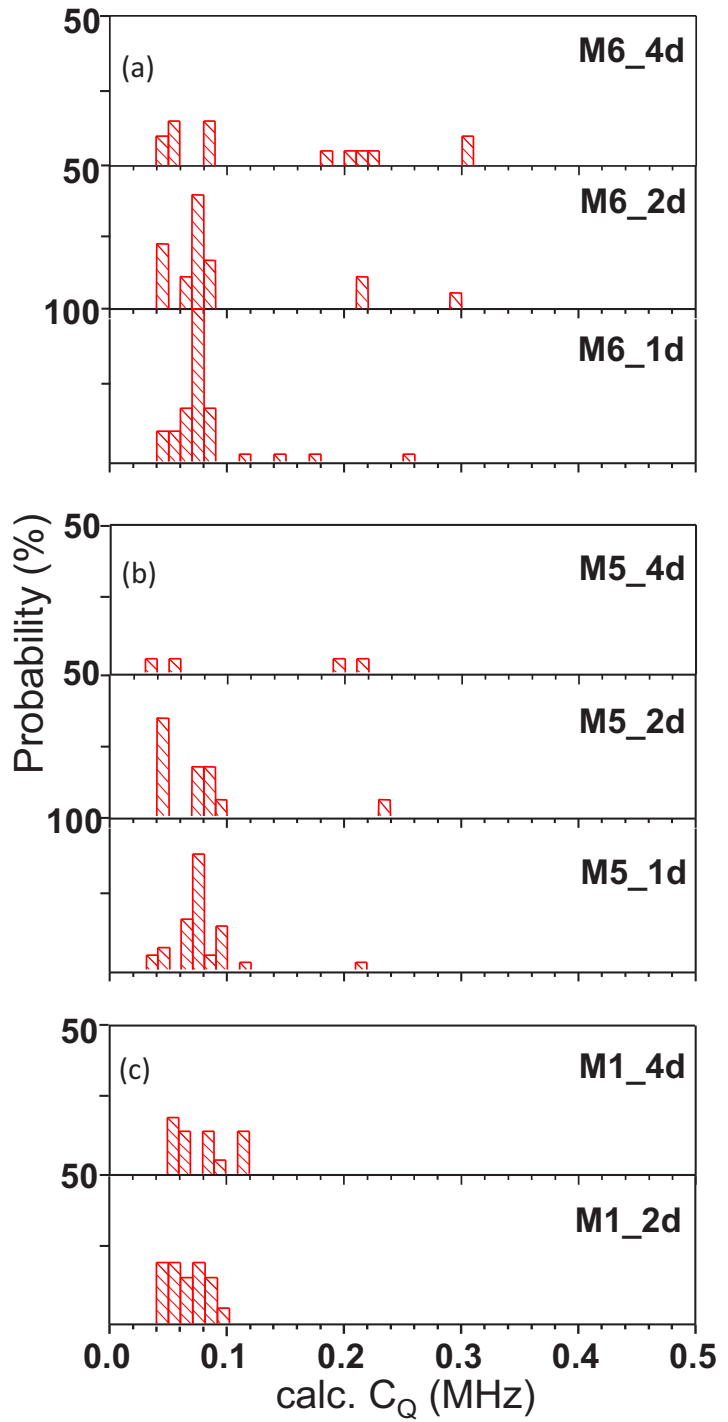

FIG. 3. Calculated $C_{Q}$ for models (a) M6, (b) M5, and (c) M1 for the defect concentrations corresponding to one (Mn-1d), two (Mn2d), and four (Mn-4d) defects in the extended supercell. Details of modeling and computation are given in Sec. II.

analyze different defect concentrations and convergence to the diluted distribution limit. The dilute defect cluster corresponds to one defect in the extended computational cell $(2 \times 2 \times 2)$, the intermediate concentration to two defects, and the dense defect concentration to four defects, denoted as Mn-1d, Mn2d, and Mn-4d, respectively. Models M1, M5, and M6 were chosen, as, on the one hand, they seem most suitable to describe the spectrum of LT-A (see the next paragraph) based on their large spread of signals and, on the other hand, they allow the incorporation of more than one defect. Figure 3 shows that the $C_{Q}$ distribution profile in the range close to $0.07 \mathrm{MHz}$ changes drastically if the defect concentration changes. This is related to a different amount of undisturbed LT visible by the dominant $C_{Q}$ contribution. It is evident that larger supercells would further increase the central contribution of defect-free LT in the fingerprints. In models M5 and M6, a minor contribution at higher $C_{Q}$ values has been found, pointing out to the presence of Li sites with larger EFGs. In model M5, these contributions
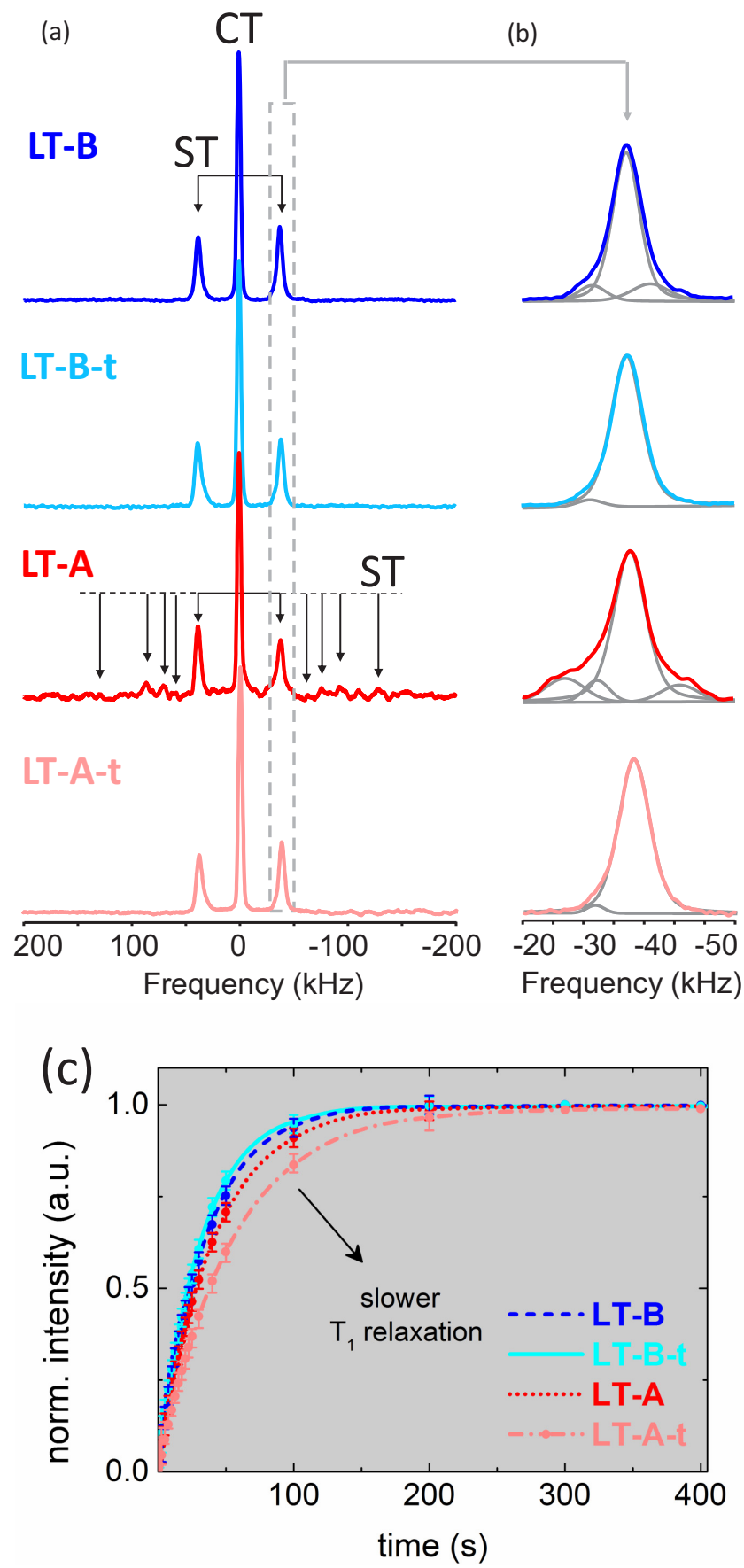

FIG. 4. (a) Broad width ${ }^{7} \mathrm{LiNMR}$ spectra of the LT samples within the spectral range of $200 \mathrm{kHz}$ to $-200 \mathrm{kHz}$. (b) The zoom-in of the selected region in (a). (c) NMR spin-lattice relaxation $T_{1}$ behavior for the LT samples.

are nearly insensitive to the defect concentration, whereas in model M6 a weak but systematic increase of the EFGs at these $\mathrm{Li}$ nuclei is visible when the concentration increases from Mn-1d to Mn-4d.

\section{B. ${ }^{7} \mathrm{Li}$ NMR}

The ${ }^{7} \mathrm{Li}$ static NMR spectra of two as-grown (LT-A and LT-B) and two thermally treated (LT-A-t and LT-B-t) lithium tantalate single crystals are presented in Fig. 4(a). Each 
TABLE II. ${ }^{7} \mathrm{Li}$ fit parameters for $\mathrm{CT}$ and the dominating ST as well as ${ }^{7} \mathrm{Li} \mathrm{CT}$ spin-lattice relaxation parameters $T_{1}^{-1}$ in LT samples.

\begin{tabular}{lccc}
\hline \hline & \multicolumn{2}{c}{${ }^{7} \mathrm{Li}$ NMR spectra } & \\
\cline { 2 - 3 } & $C_{Q}, \mathrm{kHz}$ & CT linewidth, $\mathrm{kHz}$ & $T_{1}^{-1}, 10^{-3} \times \mathrm{s}^{-1}$ \\
\hline LT-B & $77.5 \pm 0.5$ & $4.15 \pm 0.05$ & $4.8 \pm 0.2$ \\
LT-B-t & $77.5 \pm 0.5$ & $4.28 \pm 0.05$ & $4.9 \pm 0.2$ \\
LT-A & $78.5 \pm 0.5^{\mathrm{a}}$ & $3.82 \pm 0.05$ & $4.3 \pm 0.2$ \\
LT-A-t & $76.3 \pm 0.5^{\mathrm{a}}$ & $4.07 \pm 0.05$ & $3.1 \pm 0.3$ \\
\hline \hline
\end{tabular}

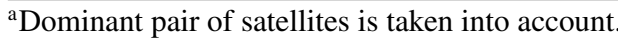

spectrum demonstrates the characteristic static single-crystal $I=3 / 2$ first-order quadrupolar line shape formed by a central line (CT) and the equally spaced satellite transitions (ST). The distance $n$ between the CT and the corresponding ST in the ${ }^{7} \mathrm{Li}$ spectrum is a measure of the quadrupolar interaction, and is related to the quadrupolar coupling constant as $C_{Q}=$ $2 I(2 I-1) \frac{v}{3}=2 v$.

Table II represents the fit parameters for the CT linewidth and the $C_{Q}$ values for the dominant pair of ST. The CT linewidth in LT-A is smaller as compared to LT-B, and the linewidth parameters increase slightly in the corresponding annealed samples.

The asymmetric ST line shapes are fitted with several components [Fig. 4(b)] indicating that the signals are composed of a number of the Li sites with different strength of quadrupolar interactions. The $C_{Q}$ values of the dominant contributions for LT-A, LT-B, and LT-B-t agree very well with the $C_{Q}$ value of $77.6 \pm 0.5 \mathrm{kHz}$ reported for an LT single crystal [26] and can be compared with 81 and $85 \mathrm{kHz}$ reported for powder samples $[25,41]$. The discrepancy observed between these samples lies within the experimental error margin and can be related to the accuracy of the angular positioning of the crystals in the magnetic field, and will therefore not be discussed here. The $C_{Q}$ in LT-A-t is remarkably lower. The spectra of LT-B and LT-B-t demonstrate only the ST signals described above, while the spectra of LT-A and LT-A-t show, in addition, a set of the reproducible minor signals, which spread across a wider range on both sides of CT. Matching a criterion of symmetry for each satellite pair relative to the CT frequency allows us to conclude that the observed signals in LT-A are the intrinsic quadrupole satellites, which belong to the signals with large and very different $C_{Q}$ parameters. Figure 2(b) represents the distribution of the experimental $C_{Q}$ values, which have been classified into the bins with a frequency width of $0.01 \mathrm{MHz}$ for LT-B and LT-B-t and 0.02 MHz for LT-A and LT-A-t. In LT-A-t, the intensities of minor signals are significantly lower and therefore cannot be quantitatively analyzed. The significant difference observed in the data from both as-grown materials points to differences in their local environments, and therefore of EFGs, which the lithium ions experience. Their structural differences as well as the effect of thermal treatment will be discussed in the next paragraph.

Measurement of the NMR relaxation rates may give independent characterization of dynamical properties of the structure and mobility associated with the different defect clusters. The experimental spin-lattice relaxation curves for all samples are shown in Fig. 4(c). Table II summarizes the rate constants (a)
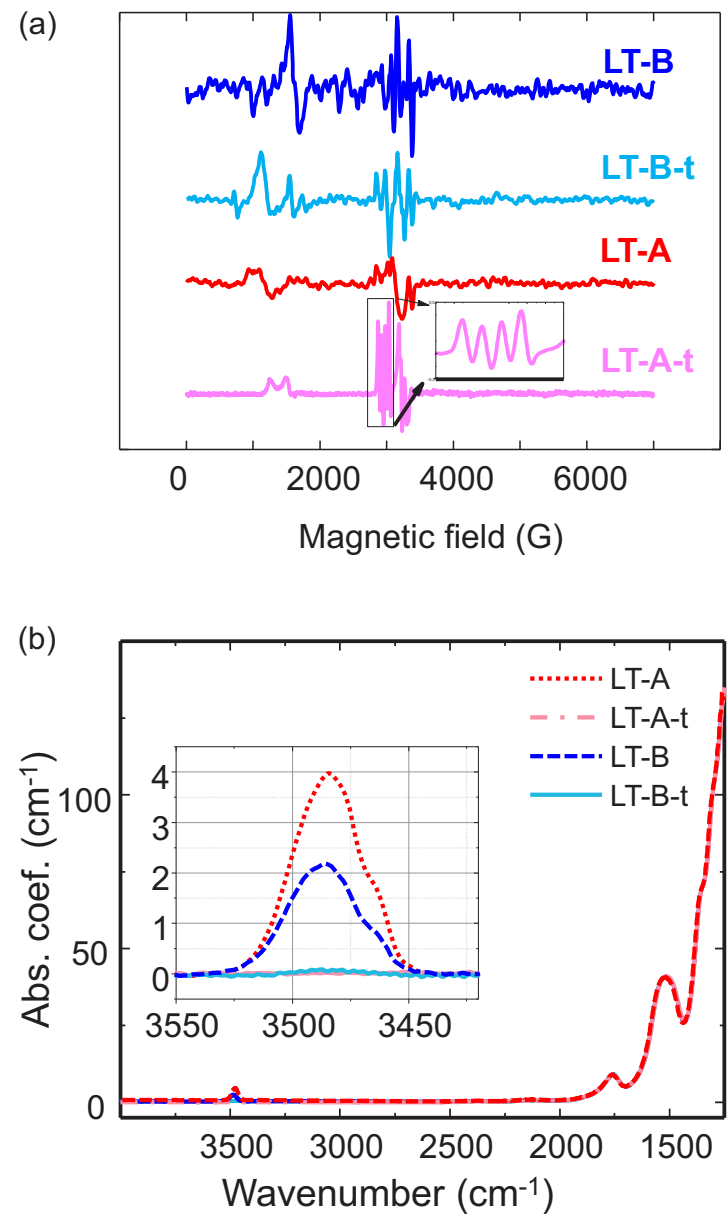

FIG. 5. (a) ESR spectra measured at the crystal $c$ axis parallel to the magnetic field, (b) FT-IR spectra of LT samples. The insert displays the zoomed region corresponding to the $\mathrm{OH}^{-}$stretching vibrations.

$T_{1}^{-1}$, which describe recovery of the magnetization along the direction of the magnetic field and quantify the rate of energy transfer from the nuclear spin system to the neighboring atoms (the lattice). Although additional information is required for deeper understanding of the relaxation behavior, our relaxation data support the observation of NMR spectroscopy, which evidences the structural difference between two congruent LT crystals of different origins.

\section{ESR}

Figure 5(a) represents the ESR spectra of the LT samples. Their complex profiles show the presence of various kinds of the paramagnetic centers in the materials under study. Based on assignments in the earlier works on congruent LT $[42,43]$, the signal at approximately $1500 \mathrm{G}$ is attributed to an unavoidable amount of paramagnetic ions such as $\mathrm{Fe}^{3+}$ as a result of the growth conditions. The signal at approximately $3300 \mathrm{G}$ is assigned to the localized electron centers associated with the crystal defects. Our measurements have not revealed any eight-line hyperfine pattern expected for $\mathrm{Ta}^{4+}$. Qualitative comparison of as-grown LT-A and LT-B shows different ESR line shape, splitting, and spectrum components 
pointing to the different origin of the paramagnetic centers in these materials. After annealing, the ESR signals at the different factors change in different ways in the samples LT-A and LT-B. In the spectrum of LT-A-t, the intensity in the $1500 \mathrm{G}$ range decreases, and four narrow lines become resolved in the region near $3000 \mathrm{G}$. This quadruplet is apparently caused by the hyperfine interaction with a ${ }^{7} \mathrm{Li}$ nuclear spin $S=3 / 2$, which proves the origin of the signal from the structural defects, such as color centers, localized charge carriers, etc. The narrow width of the lines indicates a high homogeneity of the distribution of these defects. In the spectrum of LT-B-t, the quadruplet near $3000 \mathrm{G}$ becomes also resolved with, however, much larger linewidth indicating a higher degree of disorder than in LT-A-t. The transformation of the spectrum near $1500 \mathrm{G}$ points to a less homogeneous environment around the Fe ions. For quantitative comparison, the integrated ESR intensities can be analyzed, although the spectral integration is not a straightforward way due to imprecise angular positioning of the samples. The integrated intensities normalized to the sample weight reveal the following trend: LT-A-t $<$ LT-A $<$ LT-B $<$ LT-B-t reflecting the increasing concentrations of the paramagnetic centers in the samples in this order. Thus, despite the same growth method and the same chemical composition, different defect structures have been observed in the two as-grown materials. Moreover, annealing has a prominent effect on the defect distribution.

\section{FT-infrared spectroscopy}

Figure 5(b) shows the FTIR spectra of all samples under study. In the range of $1250-1760 \mathrm{~cm}^{-1}$ the lattice vibrations have been observed. Invariance of the lattice vibration region demonstrates that the crystal lattice remains unchanged upon the thermal treatment. The absorption band consisting of two components at $3462 \mathrm{~cm}^{-1}$ and $3483 \mathrm{~cm}^{-1}$ is attributed to the $\mathrm{OH}^{-}$stretching vibrations arising from the different hydrogen binding sites [23]. Based on the melting diagram reported in Ref. [6] and the dependence of the $\mathrm{OH}^{-}$stretching band on the LT crystal composition [23,33], the nonstoichiometry of the samples under study has been determined and found to be $[\mathrm{Li}] /[\mathrm{Ta}]=48.7 / 51.3$. This value is in agreement with that published in literature for congruent LT [6]. The integrated area under the $\mathrm{OH}^{-}$band in the FT-IR spectra allows us to estimate the $\mathrm{OH}^{-}$concentration in the samples [44]. The $\mathrm{OH}^{-}$band area values indicate a higher concentration of the $\mathrm{OH}^{-}$groups in LT-A as compared to LT-B. In the annealed samples the $\mathrm{OH}^{-}$ bands almost disappear evidencing that hydrogen diffuses out of the sample as suggested in Ref. [45]. Correlation of the $\mathrm{OH}^{-}$concentration with other results will be discussed in the following section.

\section{DISCUSSION}

In order to summarize our observations, we have correlated the measured characteristics for all samples in Fig. 6. Although all involved characterization methods probe different measurands, their mutual correlation becomes meaningful and useful to generalize our findings. To specify, the NMR linewidth and spin-lattice relaxation measured on ${ }^{7} \mathrm{Li}$ are sensitive to lithium environment affected by the presence of the defects measured directly using ESR (paramagnetic defects and impurities) and

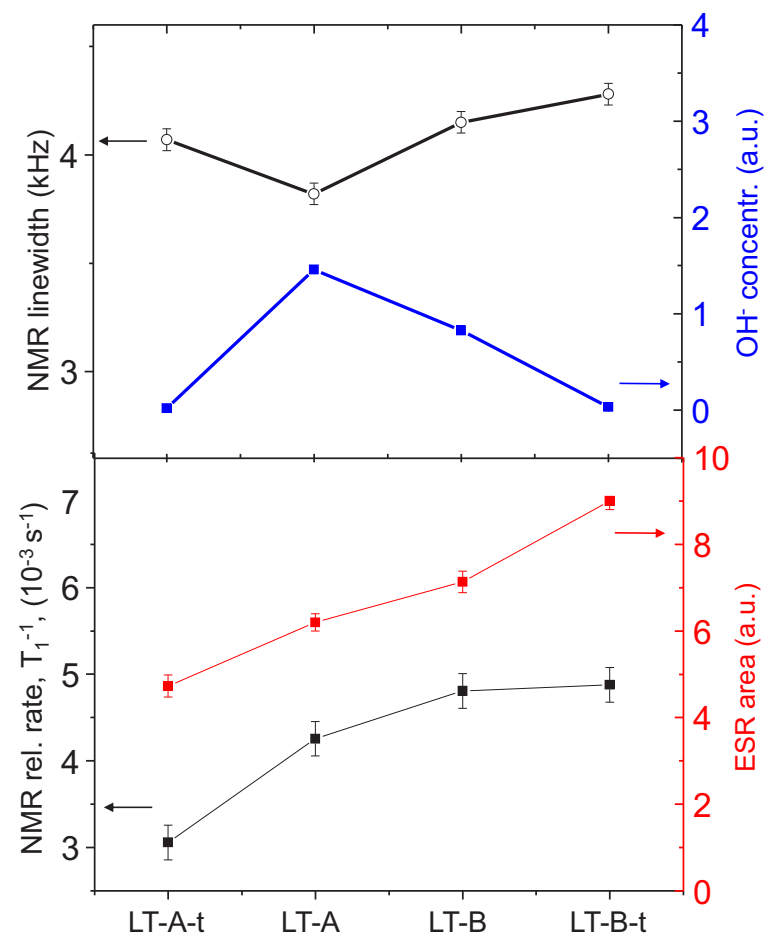

FIG. 6. Correlation of the NMR linewidth, $\mathrm{OH}^{-}$concentration determined from FT-IR data (top), NMR relaxation rate and ESR intensity (bottom).

FT-IR $\left(\mathrm{OH}^{-}\right.$defects). That is, inhomogeneous broadening of the NMR linewidth can be caused by the distribution of the local quasistatic magnetic field on the lithium sites created by the paramagnetic moments, whereas homogeneous broadening is related to dipolar interactions and molecular mobility. NMR relaxation can be facilitated by different mechanisms such as motion-induced magnetic field fluctuations, quadrupole interactions, and the presence of paramagnetic species. Integration of ESR spectra allows us to compare the concentrations of the paramagnetic centers in the samples under study. In Fig. 6, the samples have been arranged on an $x$ axis in such a way that the NMR relaxation rate $T_{1}^{-1}$ increases. This trend is accompanied by a rise of the integrated ESR intensities, which reflects an increase of the number of paramagnetic centres in the samples. However, the change in the NMR linewidth and the $\mathrm{OH}^{-}$band area follows this trend only for three samples. An opposite behavior of LT-A-t has been explained by different concentration and origin of the defects in the starting samples.

In the following sections, we discuss the observations found in this paper and divide them into three aspects: (i) effect of annealing on the structure of congruent LT; (ii) selection of the most favorable defect structure model; and (iii) structural nonidentity of two congruent LT samples.

\section{A. Annealing effect in lithium tantalate}

Based on our observations, we can suggest that the thermal treatment in lithium tantalate is accompanied by two major effects: (i) thermally induced diffusion of hydrogen, and (ii) redistribution of the defects that leads to the defect homogenization. After the heat treatment, decrease of $\mathrm{OH}^{-}$ 
concentrations has been observed in the FT-IR spectra of LT-A-t and LT-B-t. Similar observation for LT upon vacuum annealing has been reported in literature [43]. This is explained by diffusion of hydrogen out of the sample, which induces additional paramagnetic centers. Increased concentration of the defects having a magnetic moment has been observed by ESR in zirconium dioxide after annealing [46]. Concerning the defect redistribution, the ESR data indicate that the annealing process leads to a more uniform distribution of paramagnetic structural defects, especially in the sample LT-A-t. Apparently, this may indicate a more homogeneous distribution of any structural defects after annealing. The reduction of the NMR intensity of the minor signals in LT-A-t as well as disappearance of the signals with large $C_{Q}$ [Fig. 4(b)] support this scenario, because the smaller distribution of EFGs results from more uniform local environment around lithium atoms. This can be interpreted as thermally induced homogenization of the defects. Thus, we suggest the LT-A-t and LT-B-t to be the samples to compare to DFT simulations, since no hydrogen is involved in the DFT models in the present study.

\section{B. Defect structure model}

Comparing the DFT predicted and experimental classification diagrams [see Figs. 2(a) and 2(b), respectively], the most suitable defect model can be selected. The position of the dominant satellite in the experimental NMR spectra agrees well with the position of the modeled defect-free LT as guided by the dashed vertical line in Fig. 2. In principle, the experimental data reflect a superposition of the modeled defect fingerprints and the central contribution of defect-free LT. Therefore for analysis of the model, we consider the position of dominant ST and the intensity distribution of other satellites taking into account the larger contribution of defect-free sites in the realistic system. Based on our results, we suggest that the M3 model is the most favorable defect structure in the congruent LT-B-t sample after annealing. As a cross check we have compared the asymmetry parameters $\eta$ for $\mathrm{Li}$ sites with the $C_{Q}$ values close to $78 \mathrm{kHz}$. The values from DFT calculations and the orientational dependence of the NMR spectra are in a good agreement yielding $\eta$ close to 0.1 and supporting the noncentrosymmetric space group $R 3 c$ with quasiaxial symmetry of the ${ }^{7} \mathrm{Li}$ electric field gradients. Energetic calculations presented in Table I fully corroborate model M3 yielding one of the lowest formation energy values (1.61 eV) among all the models, even below that of M1 $(1.94 \mathrm{eV})$. Figure 7 provides an overview of the evaluation of the different defect models according to two criteria: (i) formation energy considerations provided by DFT, and (ii) supporting evidence from the experimental NMR data, that is $C_{Q}$ profiles. The asterisks in this evaluation diagram denote the models favored by Zotov et al. for LN according to their structural refinements of neutron and x-ray diffraction data respecting chemical reasonability of the site occupation numbers [9]. Although Zotov et al. favored M1 as the dominant defect model in LN, he also proposed M3 to give an alternative description of the nonstoichiometry in LN with the similar goodness-of-fit factors [9]. Moreover, different intrinsic defect clusters have been suggested in our previous work for lithium tantalate and lithium niobate [23].

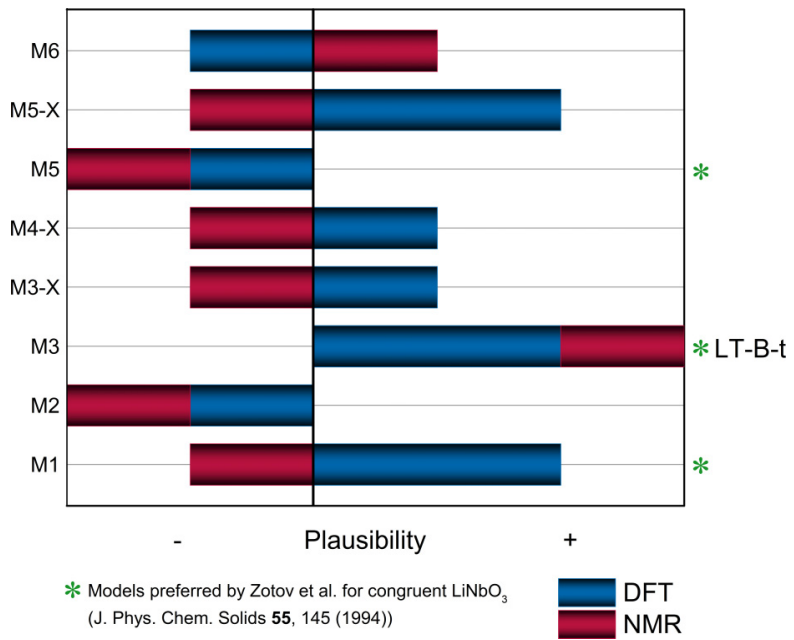

FIG. 7. Model evaluation diagram. Mn-x rows summarize all submodels within each $\mathrm{Mn}-\mathrm{x}$ family. The models are rated negatively for formation energies higher than $6 \mathrm{eV}$, positively for energies between 3 and $6 \mathrm{eV}$, and double positively for energies lower than $3 \mathrm{eV}$.

Attributing the structure of LT-A to a certain defect model is unfortunately less unambiguous. The ${ }^{7} \mathrm{Li}$ NMR spectra show additional minor signals in LT-A, which evidence the presence of $\mathrm{Li}$ sites with a great extent of electric field gradients in the sample. Hence, models M2, M5, or M6 seem reasonable to describe the defect structure in LT-A, although their formation energies are rather high. Defect formation is entropically favored at elevated temperatures, and in thermal equilibrium defect configurations with the lowest formation energies should be preferred. Nevertheless, defect structures with increased formation energies may also have certain probabilities to occur in the crystal structure. The real defect model in LT-A may be associated with a coexistence of several defect types, an inhomogeneous defect distribution (clustering), and a high concentration of the $\mathrm{OH}^{-}$groups. Based on the obvious correlation of $\mathrm{OH}^{-}$removal with diminishing the minor signals at large $C_{Q}$ after annealing [Fig. 4(a)], we suggest that the presence of $\mathrm{OH}^{-}$in the LT-A-t crystal structure is one of the origins of these signals. The detailed study of the $\mathrm{OH}^{-}$ concentration and its location in lithium tantalate by correlating DFT calculations and ${ }^{1} \mathrm{H}$ NMR is a subject for future research. First results predict that introducing $\mathrm{OH}^{-}$in the crystal lattice has a prominent effect on the distribution of the EFGs and can lead to the increase of ${ }^{7} \mathrm{Li} C_{Q}$ up to $0.55 \mathrm{MHz}$ and above. However, the absence of such signals in LT-B-despite of the intermediate $\mathrm{OH}^{-}$concentration-indicates that $\mathrm{OH}^{-}$groups are not the sole origin of the high- $C_{Q}$ signals. Indeed, a scenario of clustering has been supported by the EFG calculations for variable defect concentrations in the present work, which demonstrated increase and larger spread of EFGs at a higher concentration of defects. However, the systematic calculation of such large defect clusters to prove the aforementioned concept is not feasible due to computational limitations and complexity of the possible variations. 


\section{Structural nonidentity of congruent LT samples}

The results presented in this paper demonstrate significant differences between two congruent LT crystals, LT-A and LT-B, although grown by the same Czochralski method. Paramagnetic impurities observed in the ESR spectrum of LT-A and hydrogen defects visible in FT-IR may induce extrinsic defects and result in a great spread of electric field gradients found in the NMR spectrum of LT-A-t. The ESP data demonstrate also different origin and concentration of the paramagnetic centers in these materials that can explain significant difference in their relaxation behaviour and NMR linewidths. Thus, when characterizing the defect structure in LT, special care has to be paid to its origin, as the minor structural differences not visible by diffraction techniques, but evidenced in the present paper, can significantly affect the physical properties of the particular crystals.

\section{CONCLUSIONS}

In the present paper, we have shown feasibility of a combinational approach based on DFT and solid-state NMR to reveal the fingerprints of a specific defect configuration in congruent lithium tantalate. The electric field gradients at ${ }^{7} \mathrm{Li}$ nuclei calculated for various defect models discussed so far in literature, differ significantly. This offers the great possibility to distinguish and attribute the defect structures by means of ${ }^{7} \mathrm{Li}$ NMR spectroscopy. Using this approach, we were able to identify the intrinsic defect structure attributed to model M3 in one of two congruent lithium tantalate crystals after annealing (LT-B-t). This result is supported by the calculation of the energy for defect formation and by evaluation of the defect models by Zotov et al. [9] based on the structural refinements and chemical reasonability.
However, the defect structure in the second congruent sample (LT-A) could not be revealed based on our data. We believe that a more sophisticated defect model has to take into account extrinsic defects for balancing other impurities and/or an inhomogeneous defect distribution as well as a decoration with hydrogen. It is important to emphasize that the two congruent LT samples studied in the present work show rather different defect structures that is manifested not only in the distribution of EFGs at ${ }^{7} \mathrm{Li}$, but also in the FT-IR and ESR spectra and in the ${ }^{7} \mathrm{Li}$ spin-lattice relaxation behavior.

To sum up, the present paper has shown that there is no uniform and unique defect structure in commercially available congruent as-grown lithium tantalate crystals, but that defects vary depending on manufacturer even though the same crystal growth method has been used. The preference of a sole defect structure presumes that the samples are in thermodynamical equilibrium, which is evidently not the case for as-grown crystals without thermal treatment, as has been shown in this paper. Therefore, the vacuum annealing of samples grown by the Czochralski method is a necessary step to remove unwished impurities to ensure stable physical properties for the use in research, for applications, and for commercial devices.

\section{ACKNOWLEDGMENTS}

The authors gratefully acknowledge the financial support via the projects 'CryPhysConcept' (Grant No. 03EK3029A) and 'SyNeSteSia' (Grant No. 05K14OFA), both Federal Ministry of Education and Research (BMBF), Sächsische Aufbaubank (SAB) (Grant No. 100245339), T. Leisegang for providing the crystals and fruitful discussions, and $\mathrm{Ch}$. Irmer for technical support.
[1] F. Chen, X.-W. Wang, and K.-M. Wang, Opt. Mater. 29, 1523 (2007).

[2] V. V. Atuchin, Nucl. Instrum. Methods B 168, 498 (2000).

[3] E. Gutmann, A. Benke, K. Gerth, H. Böttcher, E. Mehner, C. Klein, U. Krause-Buchholz, U. Bergmann, W. Pompe, and D. C. Meyer, J. Phys. Chem. C 116, 5383 (2012).

[4] M. Chauvet, F. Henrot, F. Bassignot, F. Devaux, L. GauthierManuel, V. Pêcheur, H. Maillotte, and B. Dahmani, J. Opt. 18, 085503 (2016).

[5] T. Volk and M. Wöhlecke, Lithium Niobate (Springer, Berlin, 2008).

[6] S. Miyazawa and H. Iwasaki, J. Cryst. Growth 10, 276 (1971).

[7] A. Räuber, in Current Topics in Materials Science, edited by E. Kaldis (North-Holland, Amsterdam, 1978), Vol. 1, pp. 481-601.

[8] O. F. Schirmer, O. Thiemann, and M. Wöhlecke, J. Phys. Chem. Solids 52, 185 (1992).

[9] N. Zotov, H. Boysen, F. Frey, T. Metzger, and E. Born, J. Phys. Chem. Solids 55, 145 (1994).

[10] P. Lerner, C. Legras, and J. P. Dumas, J. Cryst. Growth 3-4, 231 (1968).

[11] S. Kim, V. Gopalan, K. Kitamura, and Y. Furukawa, J. Appl. Phys. 90, 2949 (2001).

[12] N. Iyi, K. Kitamura, F. Izumi, J. K. Yamamoto, T. Hayashi, H. Asano, and S. S Kimura, J. Solid State Chem. 101, 340 (1992).
[13] A. V. Yatsenko, H. M. Ivanova-Maksimova, and N. A. Sergeev, Physica B 254, 256 (1998).

[14] H. Donnerberg, S. M. Tomlinson, C. R. A. Catlow, and O. F. Schirmer, Phys. Rev. B 40, 11909 (1989).

[15] G. E. Peterson and A. Carnevale, J. Chem. Phys. 56, 4848 (1972).

[16] S. C. Abrahams and P. Marsh, Acta Cryst. B 42, 61 (1986).

[17] V. Grachev, G. Malovichko, and O. F. Schirmer, Ukr. J. Phys. 49, 438 (2004).

[18] T. Metzger, Diploma Thesis, TU München, 1993.

[19] S. C. Abrahams, J. M. Reddy, and J. L. Bernstein, J. Phys. Chem. Solids 27, 997 (1966).

[20] S. C. Abrahams, W. C. Hamilton, and J. M. Reddy, J. Phys. Chem. Solids 27, 1013 (1966).

[21] S. C. Abrahams and J. L. Bernstein, J. Phys. Chem. Solids 28 , 1685 (1967).

[22] S. C. Abrahams, W. C. Hamilton, and A. Sequeira, J. Phys. Chem. Solids 28, 1693 (1967)

[23] T. Köhler, E. Mehner, J. Hanzig, G. Gärtner, H. Stöcker, T. Leisegang, and D. C. Meyer, J Solid State Chem. 244, 108 (2016).

[24] R. K. Harris, R. E. Wasylishen, and M. J. Duer, NMR Crystallography (John Wiley \& Sons, New York, 2012).

[25] G. E. Peterson, P. M. Bridenbaugh, and P. Green, J. Chem. Phys. 46, 4009 (1967). 
[26] G. E. Peterson and P. M. Bridenbaugh, J. Chem. Phys. 48, 3402 (1968).

[27] T. K. Halstead, J. Chem. Phys. 53, 3427 (1970).

[28] J. Blümel, E. Born, and T. Metzger, J. Phys. Chem. Solids 55, 589 (1994).

[29] A. V. Yatsenko, E. N. Ivanova, and N. A. Sergeev, Physica B 240, 254 (1997).

[30] A. V. Yatsenko and E. N. Ivanova, Sov. Phys.-Solid State 37, 2262 (1995), in russian.

[31] D. C. Douglass, G. E. Peterson, and V. J. McBrierty, Phys. Rev. B 40, 10694 (1989).

[32] H. M. Petrilli, P. E. Blöchl, P. Blaha, and K. Schwarz, Phys. Rev. B 57, 14690 (1998).

[33] L. Shi, W. Yan, and Y. Kong, Eur. Phys. J. Appl. Phys. 40, 77 (2007).

[34] F. A. Kröger and H. J. Vink, in Solid State Physics, edited by F. Seitz and D. Turnbull (Academic Press, New York, 1956), p. 307.

[35] See Supplemental Material at http://link.aps.org/supplemental/ 10.1103/PhysRevMaterials.2.013804 for (1) further details of the defect cluster modeling, (2) chemical reaction equations in Kröger-Vink notation and defect formation energies calculated with DFT for different defect clusters, (3) conventions for calculating quadrupolar coupling constants, and (4) a description of the crystal orientation of LT-B and LT-B-t using a singlecrystal diffractometer.

[36] G. Kresse and D. Joubert, Phys. Rev. B 59, 1758 (1999).

[37] J. P. Perdew, K. Burke, and M. Ernzerhof, Phys. Rev. Lett. 77, 3865 (1996).

[38] G. Kresse and J. Furthmüller, Comput. Mater. Sci. 6, 15 (1996).

[39] P. Pekka, Mol. Phys. 106, 1965 (2008).

[40] D. Massiot, F. Fayon, M. Capron, I. King, S. LeCalve, B. Alonso, J.-O. Durand, B. Bujoli, Z. Gan, and G. Hoatson, Magn. Reson. Chem. 40, 70 (2002).

[41] G. Burns, Phys. Rev. 127, 1193 (1962).

[42] K. L. Sweeney, Ph.D. thesis, Oklahoma State University, 1980.

[43] C. Y. Chen, K. L. Sweeney, and L. E. Halliburton, Phys. Status Solidi A 81, 253 (1984).

[44] S. Klauer, M. Wöhlecke, and S. Kapphan, Phys. Rev. B 45, 2786 (1992).

[45] J. M. Cabrera, J. Olivares, M. Carrascosa, J. Rams, R. Müller, and E. Diéguez, Adv. Phys. 45, 349 (1996).

[46] C. Gionco, M. C. Paganini, E. Giamello, R. Burgess, C. Di Valentin, and G. Pacchioni, Chem. Mater. 25, 2243 (2013). 